\title{
On the turbulent co-spectrum of two scalars and its effect on acoustic scattering from oceanic turbulence
}

\author{
By TETJANA ROSS ${ }^{1}$, CHRIS GARRETT ${ }^{1}$ AND ROLF LUECK \\ ${ }^{1}$ Department of Physics and Astronomy, University of Victoria, Victoria, BC V8W 3P6, Canada \\ ${ }^{2}$ Centre for Earth and Ocean Research, University of Victoria, Victoria, BC V8W 2Y2, Canada
}

(Received 15 October 2003 and in revised form 20 May 2004)

While acoustic scatter from oceanic turbulence is sensitive to temperature-salinity covariations, there are unfortunately no published measurements of the turbulent temperature-salinity co-spectrum. Several models have been proposed for the form of the co-spectrum of two scalars in turbulence, but they all produce unsatisfactory results when applied to the turbulent scattering equations (either predicting negative scattering cross-sections in some regimes or predicting implausible levels of correlation between temperature and salinity at some scales). A new model is proposed and shown to give physically plausible scattering predictions in all density regimes. Highfrequency acoustic data illustrate the importance of the co-spectrum for acoustic scattering, but were collected in a density regime where there is little difference between the co-spectrum models.

\section{Introduction}

The exact form of the turbulent co-spectrum for two scalars - specifically temperature and salinity - has two key oceanographic applications. First, it is essential to making measurements of salinity microstructure (Nash \& Moum 1999, 2002). Accurate measurements of salinity microstructure and the consequent turbulent salt fluxes are an important step toward improved parameterization and predictive powers for global ocean models. As salinity cannot be measured directly, it is generally measured by proxy through conductivity measurements. But, as the conductivity of water is strongly dependent on temperature as well as salinity, the covariance between temperature and conductivity (or, equivalently, temperature and salinity) must be known in order to obtain salinity microstructure from conductivity microstructure.

Secondly, the exact form of the turbulent temperature-salinity co-spectrum is crucial to making accurate predictions of acoustic scatter from turbulent microstructure (Seim 1999). This is important because acoustic methods are showing increasing promise as a relatively cheap and easy way to make quick synoptic measurements of turbulence (Seim, Gregg \& Miyamoto 1995; Ross \& Lueck 2003; Lavery, Schmitt \& Stanton 2003).

While there has been extensive numerical modelling of the covariance (or, equivalently, the coherency) of two scalars (e.g. Yeung 1998; Fox 1999; Yeung, Sykes \& 
Vedula 2000), limitations on computational power in the direct numerical simulations have limited these studies to low Reynolds numbers and low Schmidt numbers $\left(S c=v / \kappa_{\theta}\right.$ where $v$ is the molecular viscosity and $\kappa_{\theta}$ is the molecular diffusivity of the scalar $\theta$ ). Laboratory experiments examining differential diffusion have typically been performed at either low Schmidt number (e.g. Smith et al. 1995) or low Reynolds number (Saylor \& Sreenivasan 1998). Buoyancy Reynolds numbers $\left(R e_{b}=\epsilon / \nu N^{2}\right.$, where $\epsilon$ is the rate of dissipation of turbulent energy and $N$ is the buoyancy frequency) are typically quite high when turbulent scatter is expected to be significant (the mean $R e_{b}$ is around $7 \times 10^{4}$ for our data, to be discussed later). The buoyancy Reynolds number is equivalent to the standard Reynolds number $(R e=L U / v)$ if $L$ is taken to be the scale of the largest eddy (i.e. the Ozmidov scale $\left.L_{o}=\left(\epsilon / N^{3}\right)^{1 / 2}\right)$ and $U$ is taken to be the typical scale of the fluctuating velocity (i.e. $\left.U \sim(\epsilon L)^{1 / 3}\right)$. Perhaps more importantly than the low Reynolds numbers of the experiments, however, is that the Schmidt numbers for temperature and salinity in the ocean are 8.7 and 867 , respectively, whereas at least one of the scalars had $S c \leqslant 1$ for each of the numerical experiments. When $S c \leqslant 1$, one would expect quite different physics to occur at small scales, as there is no scale separation between the smallest scales of the eddies and the diffusive scale of the scalar. Until the direct numerical simulations are able to resolve the full small-scale physics relevant to oceanic turbulence, there is a need for simple analytical models for use in the above-mentioned oceanographic applications.

The theoretical contribution of the co-spectrum to sound scattering from turbulence will be discussed in $\S 2$. This is followed in $\S 3$ by a discussion of previous models proposed for the spectrum of one scalar and the co-spectrum of two scalars with high Schmidt numbers in high Reynolds number turbulence. Section 4 introduces a revised model, which is then compared with the former models in $\S 5$. Data that demonstrate the importance of including the temperature-salinity co-spectrum in models of acoustic scatter from turbulence will also be presented in $\S 5$.

\section{Role of temperature-salinity co-spectrum in modelling of acoustic scatter from turbulence}

The basic fluid mechanics of acoustic scattering from turbulence, first introduced by Batchelor (1957), is that the turbulent motions act on the ambient gradients in sound speed and density (in the ocean these are prescribed by the temperature and salinity gradients) to create fluctuations in sound speed and density on many scales, possibly including that of the incident acoustic wave. When the incident acoustic wave encounters a parcel of water with the same density, but a different sound speed (which can be viewed as a change in density-weighted compressibility, as sound speed squared is inversely proportional to density times compressibility), the parcel of water compresses either more or less than the surrounding water and then rebounds isotropically creating a new 'scattered' sound wave. On the other hand, if the incident sound wave encounters a parcel of water with a different density, but the same sound speed, the difference in inertia between the parcel of water and the surrounding water will cause it to oscillate in the direction of the passing wave. This dipole moment creates a directional 'scattered' sound wave. A sound wave encountering a parcel of water with different sound speed and density will create a 'scattered' wave that is a combination of the above. As the sound speed and density of seawater is determined by its temperature and salinity, these fluctuations in sound speed and density are often expressed in terms of fluctuations in temperature and salinity. 
Thus, it can be shown (Batchelor 1957; Goodman 1990) that the expression for the turbulent backscattering cross-section per unit volume $\left(\sigma_{\text {turb }}\right)$ is

$$
\sigma_{\text {turb }}=2 \pi k^{4}\left(A^{2} \Phi_{T}(2 k)+B^{2} \Phi_{S}(2 k)+2 A B \Phi_{T S}(2 k)\right),
$$

where $k$ is the wavenumber of the incident acoustic wave. $\Phi_{T}, \Phi_{S}, \Phi_{T S}$ (normalized such that, for example, $\left.4 \pi \int K^{2} \Phi_{T S}(K) \mathrm{d} K=\overline{T^{\prime} S^{\prime}}\right)$ are, respectively, the isotropic vector spectra for temperature and salinity, and the temperature-salinity co-spectrum evaluated at the Bragg scattering wavelength, $2 k$. Lavery et al. (2003) showed that the coefficients are given by $A=a_{\mu}-\alpha$ and $B=b_{\mu}+\beta$, where $a_{\mu}$ and $b_{\mu}$ are, respectively, the fractional changes in sound speed from temperature and salinity changes, while $\alpha$ and $\beta$ are the coefficients of thermal expansion and saline contraction.

An examination of (2.1) reveals how the temperature-salinity co-spectrum has a large effect on the scattering cross-section. $\Phi_{T}$ and $\Phi_{S}$ are always positive, but $\Phi_{T S}$ can be either positive or negative, depending on the sign of the ratio of the ambient gradients $\delta=(\overline{\mathrm{d} T} / \mathrm{d} z) /(\overline{\mathrm{d} S} / \mathrm{d} z)$. Seim (1999) pointed out that when $\delta$ is negative, and the relative contributions to scattering from temperature and salinity are similar (he used the condition $R_{\eta}=a_{\mu} \delta / b_{\mu} \approx-1$ as he neglected density fluctuations from his model; Lavery et al. (2003) more completely propose using $R_{\rho c}=A \delta / B \approx-1$ ), the turbulent scattering signal will be greatly reduced and may disappear. Thus, for $R_{\rho c} \approx-1$, the turbulent sound scattering model is highly sensitive to the model used for the temperature-salinity co-spectrum.

\section{Previous co-spectrum models for two scalars}

As all the analytical co-spectrum models for two scalars are based on some combination of the spectra of each scalar, we start with a review of models used for spectra of a scalar $(\theta)$ in isotropic turbulence.

Dimensionally $E_{\theta}$, the wavenumber-magnitude spectrum of $\theta$ variance (normalized such that $\int E_{\theta}(K) \mathrm{d} K=\overline{\theta^{\prime 2}}$ ), is given by (Batchelor 1959)

$$
E_{\theta}(K) \propto \chi_{\theta} \epsilon^{-1 / 3} K^{-5 / 3} \quad \text { for } k_{b}<K<k_{\nu},
$$

where $\chi_{\theta}$ is the rate of dissipation of $\theta$ variance, $k_{b}=\left(N^{3} / \epsilon\right)^{1 / 2}$ is the buoyancy wavenumber and $k_{v}=\left(\epsilon / v^{3}\right)^{1 / 4}$ is the Kolmogorov wavenumber. It follows that, since $\int \Phi_{\theta}(\boldsymbol{K}) \mathrm{d} \boldsymbol{K}=\int 4 \pi K^{2} \Phi_{\theta}(K) \mathrm{d} K=\overline{\theta^{\prime 2}}$, the isotropic vector spectrum is given by

$$
\Phi_{\theta}(K)=\frac{1}{4 \pi K^{2}} E_{\theta}(K)=\frac{C}{4 \pi} \chi_{\theta} \epsilon^{-1 / 3} K^{-11 / 3} .
$$

When the Schmidt number for the scalar $\theta$ is greater than 1 (this is true for both temperature and salinity in seawater as $v \approx 1.3 \times 10^{-6} \mathrm{~m} \mathrm{~s}^{-2}, \kappa_{T} \approx 1.5 \times 10^{-7} \mathrm{~m} \mathrm{~s}^{-2}$, and $\kappa_{S} \approx 1.5 \times 10^{-9} \mathrm{~m} \mathrm{~s}^{-2}$ ), there is a viscous-convective subrange between the Kolmogorov wavenumber and the Batchelor wavenumber, $k_{B \theta}=\left(\epsilon /\left(\nu \kappa_{\theta}^{2}\right)\right)^{1 / 4}$. Then, again dimensionally,

$$
E_{\theta} \propto \chi_{\theta}(\epsilon / \nu)^{1 / 2} K^{-1} \quad \text { for } k_{v}<K<k_{B \theta} .
$$

Batchelor (1959) derived the high-wavenumber vector spectrum for a scalar in isotropic turbulence by arguing that beyond the dissipative cut-off, $k_{v}=\left(\epsilon / v^{3}\right)^{1 / 4}$, where all velocity variance is destroyed by viscosity, the distribution of $\theta$ will be affected only by the residual pure straining distortion. In a Lagrangian frame, with axes fixed to the direction of constant principal rates of strain $(\alpha, \beta, \gamma)$, this can be 
expressed as

$$
\frac{\partial \theta}{\partial t}+\alpha x \frac{\partial \theta}{\partial x}+\beta y \frac{\partial \theta}{\partial y}+\gamma z \frac{\partial \theta}{\partial z}=\kappa_{\theta} \nabla^{2} \theta .
$$

Assuming a solution of the form $\theta(x, t)=\theta_{0}(t) \mathrm{e}^{\mathrm{i} k(t) \cdot x}$, with the initial condition $\theta(t=0)=\Theta_{0} \mathrm{e}^{\mathrm{i} l \cdot x}$, we obtain

$$
\begin{aligned}
& \frac{\mathrm{d} k_{3}}{\mathrm{~d} t}=-\gamma k_{3} \quad \Rightarrow k_{3}(t)=l_{3} \mathrm{e}^{-\gamma t}, \\
& \frac{\mathrm{d} \theta_{0}}{\mathrm{~d} t}=-\kappa_{\theta} k^{2} \theta_{0} \Rightarrow \theta_{0}(t)=\Theta_{0} \exp \left[\frac{\kappa_{\theta}}{2 \alpha}\left(k_{1}^{2}-l_{1}^{2}\right)+\frac{\kappa_{\theta}}{2 \beta}\left(k_{2}^{2}-l_{2}^{2}\right)+\frac{\kappa_{\theta}}{2 \gamma}\left(k_{3}^{2}-l_{3}^{2}\right)\right] .
\end{aligned}
$$

The expressions for $k_{1}$ and $k_{2}$ are identical to (3.5), but with $\alpha$ and $\beta$ in place of $\gamma$.

After a relatively short time, the $\theta$-planes will become perpendicular to the direction of the greatest rate of contraction. In this case the greatest rate of contraction is $\gamma$, if we choose $\alpha>\beta>\gamma$. Note that $\gamma<0$, since $\alpha+\beta+\gamma=0$. Thus, defining $K=|\boldsymbol{k}|$ and $l=|\boldsymbol{l}|$,

$$
\theta \rightarrow \Theta_{0} \exp \left(\frac{\kappa_{\theta}\left(K^{2}-l^{2}\right)}{2 \gamma}\right) \mathrm{e}^{\mathrm{i} k \cdot x},
$$

for each spectral component of $\theta$. The contraction of each spectral component of $\theta$ means that $\theta$-variance is being transferred from lower wavenumbers $(l)$ to higher wavenumbers $(K)$, such that

$$
E_{\theta}(K) \frac{K}{l} \mathrm{~d} l=E_{\theta}(l) \exp \left(\frac{\kappa_{\theta}\left(K^{2}-l^{2}\right)}{\gamma}\right) \mathrm{d} l .
$$
This combined with the definition of $\chi_{\theta}$ (i.e. $\chi_{\theta}=2 \kappa \int_{0}^{\infty} K^{2} E_{\theta}(K) \mathrm{d} K$ ), leads to a
wavenumber-magnitude $\theta$-spectrum of

$$
E_{\theta}(K)=-\frac{\chi_{\theta}}{\gamma K} \exp \left(\frac{\kappa_{\theta} K^{2}}{\gamma}\right)
$$

The principle rate of contraction $(\gamma)$ is not necessarily constant throughout the fluid, however, so the true form of $E_{\theta}(k)$ is a superposition of exponentials with different exponents. Nevertheless, it is standard in the literature to approximate this superposition of exponentials as a single exponential with $\gamma \propto(\epsilon / \nu)^{1 / 2}$, where $(\epsilon / \nu)^{1 / 2}$ is the representative viscous strain rate of the turbulence. This proportionality can be expressed with the universal constant $q$ as

$$
\gamma=-\frac{1}{q}\left(\frac{\epsilon}{v}\right)^{1 / 2}
$$

where $q>0$ and the negative sign is imposed to ensure $\gamma<0$.

Kraichnan's (1968) model for the scalar spectrum, which took into consideration that strain rates may fluctuate in space and time, has a more gentle $\exp \left(-\sqrt{6 q} K / k_{B \theta}\right)$ roll-off. While some measured spectra (e.g. Nash \& Moum 2002) agree well with Kraichnan's (1968) model, others, such as the $K^{-3 / 2}$ spectra measured by Gargett (1985), fit neither theoretical form. Nevertheless, they all show a steep roll-off around the predicted wavenumber, which is all that is important for most modelling purposes. Whether a single exponential (of form $\mathrm{e}^{-x}$ or $\mathrm{e}^{-x^{2}}$ ) or a superposition of exponentials are used is therefore probably unimportant. As the physics is clearer in Batchelor's (1959) model than in Kraichnan's semi-empirical model, this paper will deal only with the Batchelor form of the spectrum. The empirically measured value of $q$ in (3.10) 
can be thought of as a factor that ensures that the roll-off occurs at the correct spot, which is what is of primary importance.

Combining (3.10) with (3.9) and (3.2), gives the isotropic high-wavenumber vector spectrum for temperature or salinity,

$$
\Phi_{\theta}(K)=\frac{q \chi_{\theta}}{4 \pi K^{3}}\left(\frac{\nu}{\epsilon}\right)^{1 / 2} \exp \left(-q K^{2} / k_{B \theta}^{2}\right) .
$$

As oceanic microstructure measurements are typically one-dimensional - collected with turbulence profilers that are either dropped vertically or towed horizontally the one-dimensional spectra, obtained through the integration

$$
\phi\left(k_{x}\right)=\iint_{-\infty}^{\infty} \Phi(\boldsymbol{k}) \mathrm{d} k_{y} \mathrm{~d} k_{z}
$$

are more relevant for comparison with data. The one-dimensional Batchelor spectrum is given by (Gibson \& Schwarz 1963)

$$
\phi_{\theta}=\frac{q \chi_{\theta}}{2 k_{x}}\left(\frac{\nu}{\epsilon}\right)^{1 / 2}\left(\exp \left(-q k_{x}^{2} / k_{B \theta}^{2}\right)-\frac{\sqrt{2 q} k_{x}}{k_{B \theta}} \int_{\frac{\sqrt{2 q q} k_{x}}{k_{B \theta}}}^{\infty} \exp \left(-x^{2} / 2\right) \mathrm{d} x\right) \text { for } k_{x}>k_{\nu} .
$$

\subsection{One-dimensional upper-bound model}

Washburn, Duda \& Jacobs (1996) and Seim (1999) applied the mathematical identity for the upper limit of a co-spectrum to the one-dimensional spectra,

$$
\phi_{T S} \leqslant\left(\phi_{T} \phi_{S}\right)^{1 / 2}
$$

to evaluate the maximum effect the co-spectrum could have.

Combining the equality in (3.14) with the Batchelor spectrum model (3.13) gives a rather complicated co-spectrum

$$
\begin{aligned}
& \phi_{T S}\left(k_{x}\right)=\frac{q\left(\chi_{T} \chi_{S}\right)^{1 / 2}}{2 k_{x}}\left(\frac{v}{\epsilon}\right)^{1 / 2} {\left[\left(\exp \left(-\frac{q k_{x}^{2}}{k_{B T}^{2}}\right)-\frac{\sqrt{2 q} k_{x}}{k_{B T}} \int_{\frac{\sqrt{2 q q} k_{x}}{k_{B T}}}^{\infty} \exp \left(-\frac{x^{2}}{2}\right) \mathrm{d} x\right)\right.} \\
&\left.\left(\exp \left(-\frac{q k_{x}^{2}}{k_{B S}^{2}}\right)-\frac{\sqrt{2 q} k_{x}}{k_{B S}} \int_{\frac{\sqrt{2 q q} k_{x}}{k_{B S}}}^{\infty} \exp \left(-\frac{y^{2}}{2}\right) \mathrm{d} y\right)\right]^{1 / 2}, \quad
\end{aligned}
$$

which, inverting (3.12), can be converted into an isotropic vector co-spectrum and then substituted into (2.1) to calculate turbulent scattering cross-sections.

This model predicts non-zero co-spectrum at low wavenumbers and zero cospectrum at higher wavenumbers (where $\phi_{T}=0$ ), and is in that way physically plausible. However, if the coherency spectrum,

$$
\gamma_{T S}=\frac{\Phi_{T S}(K)}{\left|\Phi_{T}(K)\right|^{1 / 2}\left|\Phi_{S}(K)\right|^{1 / 2}},
$$

is calculated for this model, it tends to infinity as $K / k_{B T}$ becomes large. Also, this model frequently predicts unphysical negative scattering cross-sections. These negative scattering cross-sections occur when $\phi_{T}$ becomes close to zero (i.e. when $K$ is close to $k_{B T} / \sqrt{q}$ ), for most $R_{\rho c}<0$ (note that in figure 2, to be discussed later, this occurs at $\left.\sqrt{q} K / k_{v}=\sqrt{S c q} K / k_{B T} \sim 3\right)$. 


\subsection{Co-spectrum model based on Stern's theory}

Recently Lavery et al. (2003) proposed a co-spectrum model based on Stern's (1968) theory,

$$
\phi_{T S}=\frac{1}{\delta} \phi_{T}+\frac{\kappa_{S}}{\kappa_{T}} \delta \phi_{S}
$$

where $\delta=(\overline{\mathrm{d} T} / \mathrm{d} z) /(\overline{\mathrm{d} S} / \mathrm{d} z)=\left(\chi_{T} / \chi_{S}\right)^{1 / 2}$.

Because (3.17) is linear in $\phi_{T}$ and $\phi_{S}$, the same expression applies to the vector spectra. Thus, combining (3.17) with (3.11) leads to a co-spectrum model of

$$
\Phi_{T S}=\frac{q\left(\chi_{T} \chi_{S}\right)^{1 / 2}}{4 \pi K^{3}}\left(\frac{\nu}{\epsilon}\right)^{1 / 2}\left(\exp \left(-q K^{2} / k_{B T}^{2}\right)+\frac{\kappa_{S}}{\kappa_{T}} \exp \left(-q K^{2} / k_{B S}^{2}\right)\right),
$$

which can be substituted into (2.1) to calculate turbulent scattering cross-sections.

There are several problems with this co-spectrum model. First, when $k \gg k_{B T}$ we expect no covariance between temperature and salinity (all temperature fluctuations have been damped) and, therefore, the co-spectrum must be zero. This model has a small (since $\kappa_{S} / \kappa_{T} \approx 1 / 100$ ) but finite co-spectrum for $k_{B S}>k>k_{B T}$, where we would expect no covariance. This can cause gross overestimation of the scattering crosssection at high wavenumbers when $\left|R_{\rho c}\right| \gg 1$. Second, like the previous model, the coherency spectrum (3.16) tends infinity for high $K$. The low-wavenumber limit of the coherency for this model can also be larger than 1 by $\kappa_{S} / \kappa_{T}$ (when $\delta>0$ ).

\section{New co-spectrum model}

The new co-spectrum model we propose is essentially just the application of the upper-bound model to the isotropic vector spectra rather than the one-dimensional spectra. This makes more sense physically, as the smoothing out of temperature fluctuations due to diffusion (and thus the disappearance of the co-spectrum) is more likely to be dependent on the wavenumber magnitude than the wavenumber in any particular direction (any $k_{x}$ could be made up of components with large or small wavenumber magnitude). Thus, applying the upper-bound theorem to the vector spectra, we obtain

$$
\Phi_{T S}=\left(\Phi_{T} \Phi_{S}\right)^{\frac{1}{2}}=\frac{q\left(\chi_{T} \chi_{S}\right)^{1 / 2}}{4 \pi K^{3}}\left(\frac{\nu}{\epsilon}\right)^{1 / 2} \exp \left(-q K^{2} / k_{B T S}^{2}\right),
$$

where

and thus,

$$
\frac{1}{k_{B T S}^{2}}=\frac{1}{2}\left(\frac{1}{k_{B T}^{2}}+\frac{1}{k_{B S}^{2}}\right)=\frac{1}{2}\left(\frac{\nu}{\epsilon}\right)^{1 / 2}\left(\kappa_{T}+\kappa_{S}\right)
$$

$$
k_{B T S}=\left\{\epsilon /\left[v\left(\frac{\kappa_{T}+\kappa_{S}}{2}\right)^{2}\right]\right\}^{1 / 4}
$$

could be considered the 'Batchelor wavenumber' of the co-spectrum. As before, (4.1) can be substituted into (2.1) to predict acoustic scattering cross-sections.

\subsection{Direct derivation of a 'Batchelor' co-spectrum}

Looking back to the derivation of the scalar spectrum (3.11), we can argue that (4.1) is more than just an upper limit for the co-spectrum, but is an exact solution for isotropic turbulence. Note, in (3.5), that the wavenumber depends only on the 
background straining rate $\gamma=-(\epsilon / v)^{1 / 2} / q$, which will be the same for all scalars. So, analogously to Batchelor (1959) argument, we can write

$$
E_{T S}(K) \frac{K}{l} \mathrm{~d} l=E_{T S}(l) \exp \left(\frac{\kappa_{T}\left(K^{2}-l^{2}\right)}{2 \gamma}\right) \exp \left(\frac{\kappa_{S}\left(K^{2}-l^{2}\right)}{2 \gamma}\right) \mathrm{d} l,
$$

and, assuming

derive (4.1) directly.

$$
\int_{0}^{\infty} K^{2} E_{T S}(K) \mathrm{d} k=\left(\frac{\chi_{T}}{2 \kappa_{T}} \frac{\chi_{S}}{2 \kappa_{S}}\right)^{1 / 2}
$$

Another way to look at it is that, because the time dependence of the wavenumber is not scalar-dependent, $T$ and $S$ will remain phase locked as they evolve to higher wavenumbers and, although the co-spectrum will eventually disappear as $T$ fluctuations are diffused away, the coherency between $T$ and $S$ will remain 1 . With perfect coherency, $\Phi_{T S}(K)=\left(\Phi_{T}(K) \Phi_{S}(K)\right)^{1 / 2}$ is exact.

This assumption of perfect coherency throughout the whole of wavenumber space may not prove to be justified. If the true scalar spectra and co-spectra were a superposition of exponentials with different $\gamma$, there would be a loss of coherency (with a superposition of even two exponentials, the 'Batchelor derived' co-spectrum is no longer equivalent to the upper-bound (4.1) thereby reducing the coherency).

Direct numerical simulations with low Schmidt and Reynolds numbers (Yeung et al. 2000) and low Reynolds number laboratory experiments (Saylor \& Sreenivasan 1998) have shown that there is a loss of coherency between scalars beyond the Batchelor wavenumber of the more diffusive scalar, and that these double-diffusive effects can even 'backscatter' to lower wavenumbers. Based on their numerical results, Yeung et al. (2000) predict that this 'backscattering' to lower wavenumbers will become more important at large Schmidt number. Another view, however, is that, for larger Schmidt numbers, the larger separation between the energy-containing wavenumbers and the wavenumbers at which diffusion is important will render this effect negligible (in terms of Yeung et al.'s (2000) triad interactions this means that the wavenumbers of the two scalar modes become even larger relative to the velocity mode connecting them - so the 'backscatter' only affects very high wavenumbers). The laboratory experiments of Saylor \& Sreenivasan (1998) found evidence of differential diffusion at scales much larger than the Batchelor scale for scalars with very large Schmidt numbers $(S c=1200$ to 77000$)$. Thus, while the predictions of Yeung et al. (2000) have their foundation in high-Sc laboratory results, the experiments were conducted at low Reynolds number, so it is unclear whether their results can be extrapolated to high Re.

Further study is necessary to determine the true form of the coherency spectrum at high Schmidt and Reynolds numbers. Until such a time, we argue that it is better to assume perfect coherency, than to use one of the previous models, where the coherency tends to infinity for high $K$. In the context of the acoustic scattering model, any loss of coherency beyond the roll-off of the temperature spectrum should make very little difference, as one would simply be multiplying a number of order 1 by a vanishingly small temperature spectrum.

As with the one-dimensional upper-bound model, this three-dimensional upperbound model predicts a plausible co-spectrum for low wavenumbers, which then goes to zero at higher wavenumbers (i.e. when $\Phi_{T} \rightarrow 0$ ). Additionally, with this model $\sigma_{\text {turb }}$ cannot be negative (since $\Phi_{T}$ and $\Phi_{S}$ are positive definite, $\left(A \sqrt{\Phi_{T}} \pm B \sqrt{\Phi_{S}}\right)^{2} \geqslant 0$ ), making this model at least physically plausible for all combinations of parameters. 

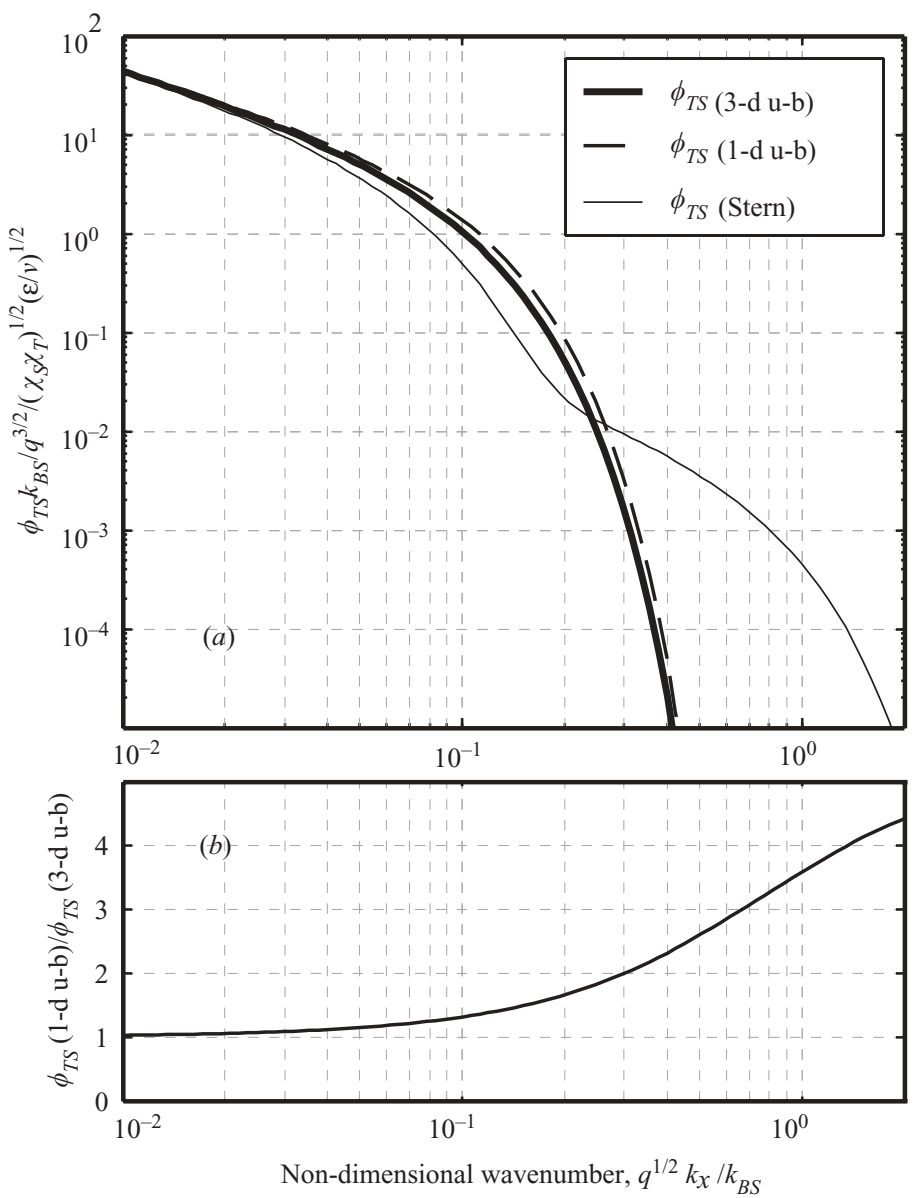

FIGURE 1. Comparison of different one-dimensional temperature-salinity co-spectrum models, using Batchelor scalar spectra. The ratio of the one-dimensional to the three-dimensional upper-bound co-spectrum shown in $(b)$ brings out differences which are more difficult to see in the logarithmic plot $(a)$.

This three-dimensional upper-bound co-spectrum also yields a much simpler expression for the one-dimensional co-spectrum,

$$
\begin{aligned}
& \phi_{T S}\left(k_{x}\right)=\frac{q\left(\chi_{T} \chi_{S}\right)^{1 / 2}}{2 k_{x}}\left(\frac{v}{\epsilon}\right)^{1 / 2}\left(\exp \left(-\frac{q k_{x}^{2}}{k_{B T S}^{2}}\right)-\frac{\sqrt{2 q} k_{x}}{k_{B T S}} \int_{\frac{\sqrt{2} q k_{x}}{\bar{k}_{B T S}}}^{\infty} \exp \left(-\frac{x^{2}}{2}\right) \mathrm{d} x\right) \\
& \text { for } k_{x}>k_{\nu} \text {. }
\end{aligned}
$$

Note that (4.3) is directly analogous to the Batchelor spectrum for a scalar (3.13), as opposed to the much more complicated one-dimensional version (3.15).

\section{Comparing co-spectrum models}

Figure 1 shows the different forms of the co-spectra for the scalars temperature and salinity in turbulent seawater (i.e. with Lewis number $L e=\kappa_{T} / \kappa_{S}=100$ ). The Stern model diverges quite significantly from the other two models and, as mentioned earlier, predicts far too much variance at wavenumbers beyond the diffusive cut-off 

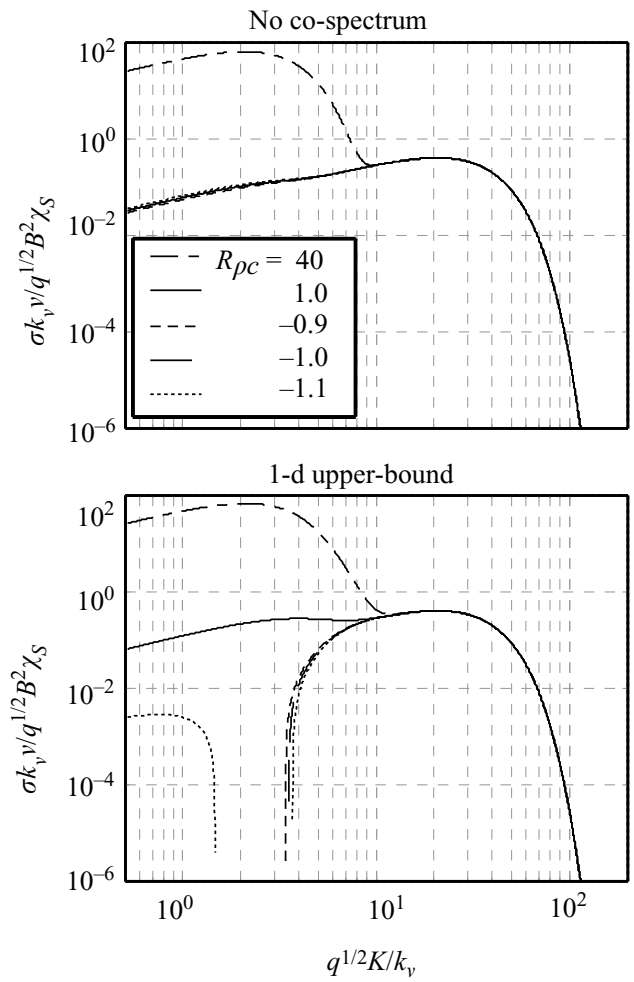
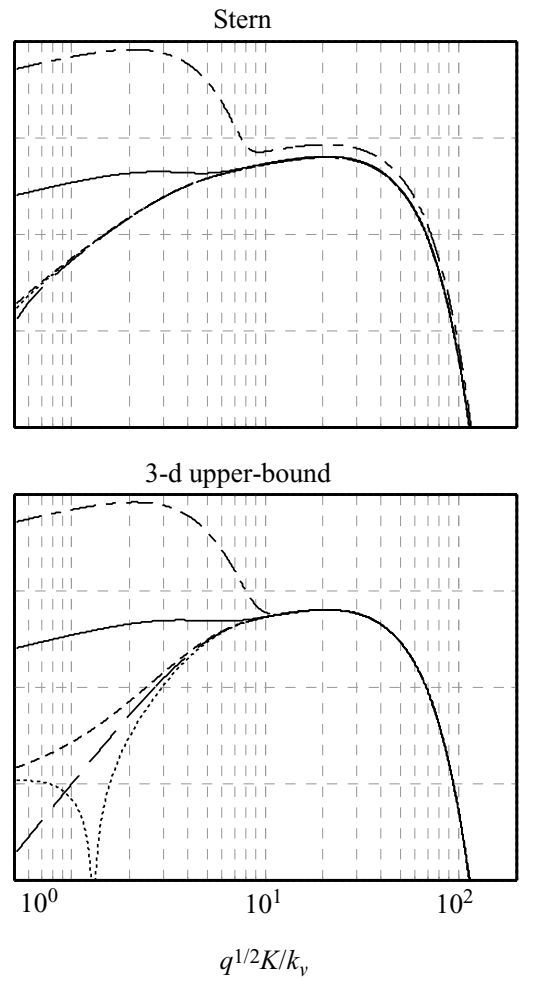

FiguRE 2. Comparison of scattering models for different co-spectrum models, calculated from (2.1) using (3.11) and the co-spectrum models detailed in $\S \S 3.1,3.2$ and 4 . When $\left|R_{\rho c}\right|$ is close to 1 , salinity and temperature contribute roughly equally to sound scattering from turbulence and scattering is much reduced at low wavenumbers, where they cancel. Both the scattering cross-section $(\sigma)$ and the Bragg wavenumber $(K=2 k$ where $k$ is the wavenumber of the incident acoustic wave) have been non-dimensionalized, such that the shape of the scattering curve is determined by $R_{\rho c}$, and the Schmidt numbers $v / \kappa_{S}$, and $v / \kappa_{T}$. The missing portions of the curves are where the models predicted negative scattering cross-sections. The scattering cross-section is only calculated for $K>(5 / 12)^{3 / 2} k_{v}$, as below that is the inertial-convective regime (Seim 1999), which is not modelled here. A value of $q=3.7$ (Oakey 1982) was used to determine the position of the cut-off on the scaled wavenumber axis.

for heat. The differences between the two upper-bound models are not large but increase as the wavenumber approaches the diffusive cut-off for salt $\left(k_{B S}\right)$. Measurements such as those performed by Nash \& Moum (2002) may be able to detect the difference between the two upper-bound models, but unfortunately there are currently no published oceanic temperature-salinity co-spectra data with which to compare the models.

\subsection{Applied to the acoustic model}

For most values of $R_{\rho c}$, the effect that the different co-spectrum models have on the acoustic scattering model is negligible. In certain circumstances, however, such as when $R_{\rho c} \approx-1$ or $\left|R_{\rho c}\right|$ is large (see figure 2 ), there can be large differences between the models.

In figure 2, note that the Stern model and both upper-bound models give essentially identical results for $R_{\rho c}=1$. When $\left|R_{\rho c}\right| \gg 1$, however, the Stern model significantly over- (or under-, if $R_{\rho c}<0$ ) predicts the scattering cross-section for large $K$. This 
is a consequence of the fact that it erroneously predicts a small but finite cospectrum beyond the diffusive cut-off for temperature. Also note that when $R_{\rho c} \approx-1$, the one-dimensional upper-bound model gives negative scattering cross-sections for $0.2<q^{1 / 2} k / k_{v}<2$, and while the three-dimensional upper bound model cannot give negative cross-sections, it does go to zero for $R_{\rho c}<-1$.

\section{2. $44.7 \mathrm{kHz}$ sounder data illustrates importance of including co-spectrum}

In a field study in Knight Inlet in June 2001 (Ross \& Lueck 2003) we collected co-located acoustic and microstructure data. There were two sounders mounted on a horizontally towed microstructure instrument. While the acoustic data collected at $307.2 \mathrm{kHz}$ are discussed at length in Ross \& Lueck (2003), here we present $44.7 \mathrm{kHz}$ data that illustrate the importance of including the co-spectrum when modelling acoustic scatter from turbulence.

Temperature gradient microstructure is visually correlated with acoustic scatter at $44.7 \mathrm{kHz}$ (figure 3). While this does not in itself constitute proof that the scatter is from turbulence, in Ross \& Lueck (2003) we did a careful analysis of the stronger returns seen in the $307.2 \mathrm{kHz}$ data, concluding that they could not be biological and were a good fit to the scattering model. As the animals collected in net-hauls in the turbulent region were generally smaller than the wavelength of $44.7 \mathrm{kHz}$ sound, the bioacoustic signal should be even less of a confounding factor at $44.7 \mathrm{kHz}$. Therefore, it is safe to assume that the scatter seen in figure 3 is from turbulence.

While the $44.7 \mathrm{kHz}$ instrument we used could not be calibrated to better than $\pm 5 \mathrm{~dB}$, the turbulence scattering data still show that the co-spectral contribution to scattering is definitely non-negligible (note that, in figure 4, the line neglecting the cospectrum does not overlap the $44.7 \mathrm{kHz}$ data points). This is because temperature and salinity are negatively correlated (on average $R_{\rho c}=-0.75$ ) in Knight Inlet, and thus, the effect of neglecting the temperature-salinity co-spectrum is to overestimate the volume scattering strength by $12 \mathrm{~dB}$ (or, in linear space, overestimate the scattering cross-section by a factor of 16). The volume scattering strengths predicted while neglecting the co-spectrum were in fact $17 \mathrm{~dB}$ higher than the measured $S v$ (i.e. the predicted $\sigma$ was 50 times larger than what was measured).

Neglecting the co-spectrum has little effect on the $307.2 \mathrm{kHz}$ data (figure 4), because most of the data were collected when the turbulent conditions were such that temperature variations (and hence temperature-salinity co-variations) at the wavelength of $307.2 \mathrm{kHz}$ sound were being smoothed away due to diffusion.

While the $44.7 \mathrm{kHz}$ data show that the co-spectrum cannot be neglected in the modelling of sound scatter from turbulence, they are not of good enough quality to distinguish between the Stern and three-dimensional upper-bound models (the onedimensional upper-bound model predicts negative $\sigma_{t u r b}$, and so is obviously incorrect in this density regime). While the spread in the $307.2 \mathrm{kHz}$ data is smaller, because of where they fall on the spectrum of turbulent scattering cross-sections (figure 4), they also offer no help in identifying the correct co-spectrum model (aside from also rejecting the no-correlation and one-dimensional upper-bound models).

Another consideration in the interpretation of these acoustic data is Gargett's (1985) careful study of turbulent temperature spectra in Knight Inlet (very near the location where our acoustic data were collected). This study found that $\Phi_{T}(K)$ only followed the classical form - (3.1), followed by (3.9) with $q \sim 4-$ when the parameter $I=k_{v} / k_{b}=\left(R e_{b}\right)^{3 / 4}$ was around 50-100 (labelled Class B). When $I$ was larger (up to $O(3000)$, labelled Class A), $E_{T}(K)$ followed a $K^{-3 / 2}$ scaling throughout the inertialconvective (classically $K^{-5 / 3}$ ) and viscous-convective (classically $K^{-1}$ ) subranges, 


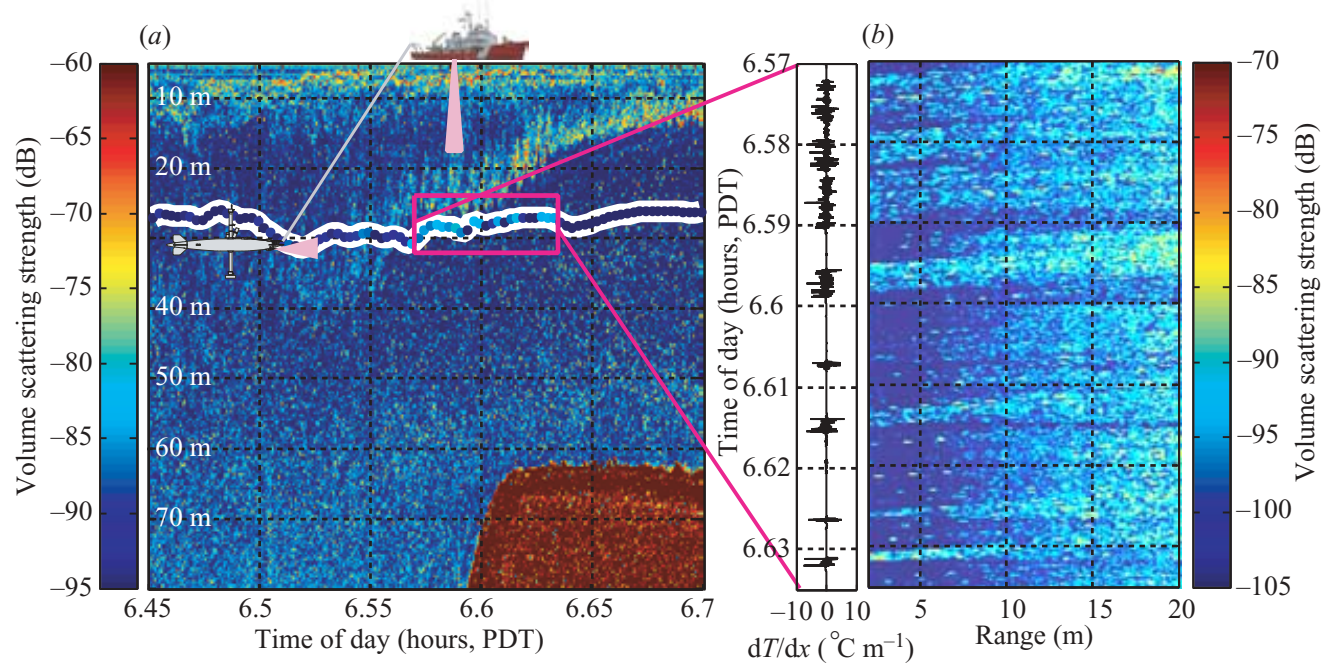

Figure 3. (a) An echogram from the $100 \mathrm{kHz}$ ship-board sounder. The white line is the approximate path of the towed vehicle (the ship-board sounder data are lagged to line up with vehicle data) and the overlying coloured circles are predicted backscatter at $100 \mathrm{kHz}$ as estimated from microstructure data. (b) Echogram from the $44.7 \mathrm{kHz}$ vehicle-mounted sounder for time and depth of the pink box in $(a)$. Each horizontal line shows the echo from one ping. As time progresses downwards and the range is the distance ahead of the vehicle, parcels of water travel diagonally across the figure, from right to left. The temperature-gradient microstructure is shown on the left.

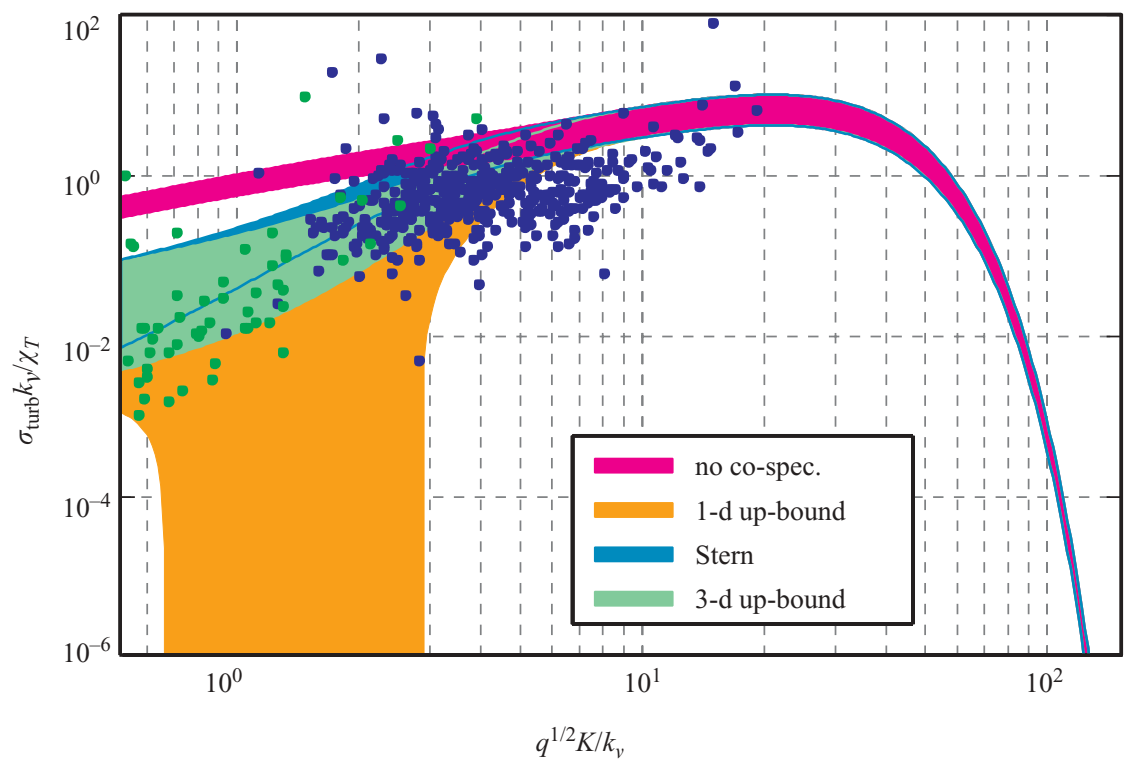

FiguRE 4. Wavenumber spectra of scaled turbulent scattering cross-sections for different co-spectrum models. The points are measured scattering cross-section (green $44.7 \mathrm{kHz}$, blue $307.2 \mathrm{kHz}$ ), which are scaled by $k_{\nu} / \chi_{T}$ to collapse them onto the same model. The thickness of the spectra allow for the range of $R_{\rho c}$ measured when there were acceptable echoes at 44.7 $\mathrm{kHz}\left(R_{\rho c}=-0.71 \pm 0.15\right)$. Data are only presented for $K>(5 / 12)^{3 / 2} k_{v}$ - beyond the transition to the viscous-convective subrange, and $q=3.7$ was used to scale the wavenumber axis. 
followed by a Batchelor-like exponential decay with $q \sim 12$. As our turbulent scattering measurements were collected in regions of high $R_{e}$, most of the data in figure 4 fall into the non-traditional Class A. Goodman (1990) has discussed the effect that this alternative spectral shape would have on acoustic scattering for the case of temperature microstructure. If we were to substitute an empirical Class A model for the traditional Batchelor model (3.11) in (2.1), there would be no substantial difference to our conclusions vis-à-vis the effect of the co-spectrum. The different $q$ would shift the region of negative scattering cross-sections to lower $K / k_{v}$, and also decrease the wavenumber at which salinity scatter begins to dominate. As the level of a Class A spectrum is higher at low wavenumber, the effect of using Class A spectra would be to increase the predicted total level of scatter at low wavenumbers. This would decrease the agreement currently found in figure 4 , as the traditional spectra model already slightly overestimates the turbulent scatter.

\section{Conclusions}

A new co-spectrum model for two scalars in high Schmidt and Reynolds number turbulence was introduced here with a view to applications in the ocean. This model can be viewed either as the upper bound for an isotropic vector co-spectrum or as an extension to Batchelor's (1959) derivation of the spectrum of a scalar in turbulence. Its fundamental assumption of perfect coherency between the scalars at all wavenumbers needs to be tested through high $S c$ and Re numerical and/or laboratory experiments.

While there are no published oceanic turbulent scalar co-spectra data to compare this and previous models with, this model is shown to be simpler and more physically plausible than those used in previous oceanographic literature. It also functions much better in models of acoustic scatter from oceanic turbulence, which are shown to be sensitive to the inclusion of a reasonable temperature-salinity co-spectrum model.

Given the sensitivity of the scattering models to the co-spectrum model used, it would be interesting to see samples from regions with $R_{c \rho} \approx-1$, as it is there that we predict the biggest discrepancies between the scattering predictions based on the different temperature-salinity co-spectra.

We thank the reviewers for instructive comments. We thank ASL Environmental Sciences for the loan of the echosounders. This work was supported by NSERC and by ONR under grant \#N00014-93-1-0362.

\section{REFERENCES}

Batchelor, G. K. 1957 Wave scattering due to turbulence. In Proc. Symp. on Naval Hydrodynamics, pp. 409-423. National Academy of Sciences, Washington, DC.

BAtCHELOR, G. K. 1959 Small-scale variation of convected quantities like temperature in turbulent fluid. J. Fluid Mech. 5, 113-139.

Fox, R. O. 1999 The Lagrangian spectral relaxation model for differential diffusion in homogeneous turbulence. Phys. Fluids 11, 1550-1571.

GARGETT, A. E. 1985 Evolution of scalar spectra with the decay of turbulence in a stratified fluid. J. Fluid Mech. 159, 379-407.

Gibson, C. H. \& Schwartz, W. H. 1963 The universal equilibrium spectra of turbulent velocity and scalar fields. J. Fluid Mech. 16, 365-384.

Goodman, L. 1990 Acoustic scattering from ocean microstructure. J. Geophys. Res. 95, 11,55711,573 .

Kraichnan, R. 1968 Small-scale structure of a scalar field convected by turbulence. Phys. Fluids 11, 945-953. 
Lavery, A. C., Schmitt, R. W. \& Stanton, T. K. 2003 High-frequency acoustic scattering from turbulent oceanic microstructure: the importance of density fluctuations. J. Acoust. Soc. Am. 114, 2685-2697.

NAsh, J. D. \& Moum, J. N. 1999 Estimating salinity variance dissipation rate from microstructure measurements. J. Atmos. Ocean. Tech. 16, 263-274.

Nash, J. D. \& Moum, J. N. 2002 Microstructure estimates of turbulent salinity flux and the dissipation spectrum of salinity. J. Phys. Oceanogr. 32, 2312-2333.

OAKEY, N. S. 1982 Determination of the rate of dissipation of turbulent energy from simultaneous temperature and velocity shear microstructure measurements. J. Phys. Oceanogr. 12, 256-271.

Ross, T. A. R. \& Lueck, R. G. 2003 Sound scatter from oceanic turbulence. Geophys. Res. Lett. 30, 1344, doi:10.1029/2002GL016733.

Saylor, J. R. \& SReenivasan, K. R. 1998 Differential diffusion in low Reynolds number water jets. Phys. Fluids 10, 1135-1146.

SeIm, H. E. 1999 Acoustic backscatter from salinity microstructure. J. Atmos. Ocean. Tech. 16, $1491-1498$.

Seim, H. E., Gregg, M. C. \& Miyamoto, R. T. 1995 Acoustic backscatter from turbulent microstructure. J. Atmos. Ocean. Tech. 12, 367-380.

Smith, L. L., Dibble, R. W., Talbot, L., Barlow, R. S. \& Carter, C. D. 1995 Laser Raman scattering measurements of differential diffusion in nonreacting turbulent jets of $\mathrm{H}_{2} / \mathrm{CO}_{2}$ mixing with air. Phys. Fluids 7, 1455-1466.

Stern, M. E. 1968 T-S gradients on the micro-scale. Deep-Sea Res. 15, 245-250.

WAShburn, L., DudA, T. F. \& JACOBS, D. C. 1996 Interpreting conductivity microstructure: estimating the temperature variance dissipation rate. J. Atmos. Ocean. Tech. 13, 1166-1188.

Yeung, P. K. 1998 Correlations and conditional statistics in differential diffusion: Scalars with uniform mean gradients. Phys. Fluids 10, 2621-2635.

Yeung, P. K., Sykes, M. C. \& Vedula, P. 2000 Direct numerical simulation of differential diffusion with Schmidt numbers up to 4.0. Phys. Fluids 12, 1601-1604. 